\title{
Independence and matching numbers of unicyclic graphs from null space
}

\author{
L. Emilio Allem ${ }^{1}$. Daniel A. Jaume ${ }^{2}$. Gonzalo Molina ${ }^{2} \cdot$ Maikon M. Toledo $^{1}$. \\ Vilmar Trevisan ${ }^{1}$
}

Received: 12 February 2019 / Revised: 11 November 2019 / Accepted: 21 December 2019 /

Published online: 11 February 2020

(c) SBMAC - Sociedade Brasileira de Matemática Aplicada e Computacional 2020

\begin{abstract}
We characterize unicyclic graphs that are singular using the support of the null space of their pendant trees. From this, we obtain closed formulas for the independence and matching numbers of a unicyclic graph, based on the support of its subtrees. These formulas allows one to compute independence and matching numbers of unicyclic graphs using linear algebra methods.
\end{abstract}

Keywords Unicyclic · Null space · Nullity · Support · Independence number · Matching number

Mathematics Subject Classification $05 \mathrm{C} 50 \cdot 15 \mathrm{~A} 18$

\section{Introduction}

Recently, in Jaume and Molina (2018), the authors studied the null space of the adjacency matrix of trees and they presented a null decomposition of trees. In general, this null decomposition divides a tree into two forests (one of the forests can be empty), one composed by

Communicated by Maria Aguieiras de Freitas.

\section{Emilio Allem}

emilio.allem@ufrgs.br

Daniel A. Jaume

djaume@unsl.edu.ar

Gonzalo Molina

lgmolina@unsl.edu.ar

Maikon M. Toledo

maikon.toledo@ufrgs.br

Vilmar Trevisan

trevisan@mat.ufrgs.br

1 Instituto de Matemática, UFRGS-Universidade Federal do Rio Grande do Sul, Porto Alegre, Brazil

2 Departamento de Matemáticas, Universidad Nacional de San Luis, San Luis, Argentina 
singular trees and the other composed by non-singular trees. The technique used was the analysis of the support of the tree, where the support is defined as the subset of vertices for which at least one of its corresponding coordinates of the eigenvectors of the null space of the adjacency matrix is nonzero.

As an application, in Jaume and Molina (2018), the null decomposition was used to obtain closed formulas for two classical parameters. The first one is the independence number of a graph $G$, denoted by $\alpha(G)$. Notice that the problem of computing $\alpha(G)$ is NP-hard Karp (1972) and several mathematicians have studied $\alpha(G)$ (for example, Alon and Kahale 1998; Frieze 1990; Shearer 1983). The second one is the matching number of a graph $G$, denoted by $v(G)$ (Cvetkovć et al. 1980; Ming and Wang 2001). Historically, the matching theory started with bipartite graphs and one of the earliest works was published in 1916 (König 1916).

In this paper, we extend the results of Jaume and Molina (2018) to unicyclic graphs. In a more general sense, we obtain structural information of the unicyclic graphs using the support of their subtrees. In particular, we obtain closed formulas for the independence and matching numbers of unicyclic graphs that depend on the support and the core of their subtrees. Next we give an outline of this paper. It is worth pointing out that, in practice, this means that these classical parameters can be computed using linear algebra.

In Sect. 2, we present some basic notations and definitions of support of a graph. In Sect. 3, we characterize singular unicyclic graphs using the support of their pendant trees. In Sect. 4, we obtain a closed formula for the independence number of unicyclic graphs using the support of the subtrees of these unicyclic graphs. In Sect. 5, we obtain closed formulas for the matching number of unicyclic graphs based on the support of subtrees of these unicyclic graphs.

\section{Basic definitions and notation}

In this section, we present some notation and basic definitions. In particular, we explain the notion of support of a graph. We use the graphs of Fig. 1 to illustrate the concepts used here.

Let $G=(V, E)$ be a simple graph of order $n$, with vertex set $V=\left\{v_{1}, \ldots, v_{n}\right\}$ and edge set $E=E(G)$, the adjacency matrix $A(G)=\left(a_{i j}\right)_{n \times n}$ of $G$ is defined as

$$
a_{i j}= \begin{cases}1, & \text { if }\left\{v_{i}, v_{j}\right\} \in E ; \\ 0, & \text { if }\left\{v_{i}, v_{j}\right\} \notin E .\end{cases}
$$

Denote by $\varepsilon_{\lambda}$ the $\lambda$-eigenspace of $A(G)$; thus, $\varepsilon_{\lambda}=\left\{x \in \mathbb{R}^{n}: A(G) x=\lambda x\right\}$. The 0 eigenspace $\left(\varepsilon_{0}\right)$ is the focus of our work and will be denoted by $\mathcal{N}(G)$. The nullity of a graph $G$, denoted by $\eta(G)$, is the multiplicity of the eigenvalue zero in the spectrum of $A(G)$ or, equivalently, the dimension of the 0 -eigenspace of $G$. The graph $G$ is called singular if $A(G)$ is a singular matrix or $\eta(G)>0$. Otherwise, the graph $G$ is called nonsingular.

As an example, we observe that the set $S=\left\{(0,1,0,-1,0,0)^{t},(0,0,1,-1,0,0)^{t}\right\}$ is a basis for the null space of the tree $T_{1}$ of Fig. 1 ; hence, $\eta\left(T_{1}\right)=2$. And we notice that 0 is not an eigenvalue of the tree $T_{2}$ of Fig. 1 , so $\eta\left(T_{2}\right)=0$.

Definition 2.1 A set $I \subset V$ of vertices of a graph $G$ is an independent set in $G$ if no two vertices in $I$ are adjacent. A maximum independent set is an independent set of maximum cardinality. The cardinality of any maximum independent set in $G$, denoted by $\alpha(G)$, is called the independence number of $G . \mathcal{I}(G)$ denotes the set of all maximum independent sets of $G$. 


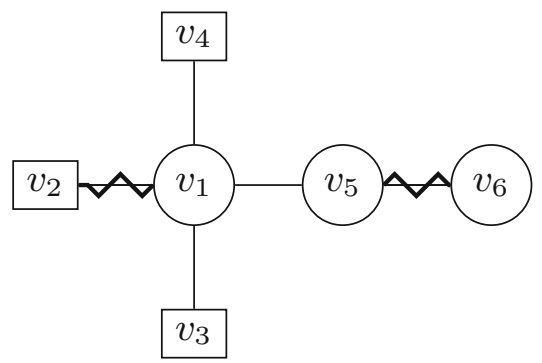

$T_{1}$

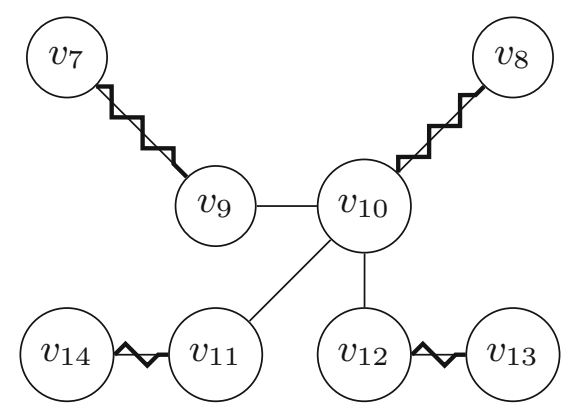

$T_{2}$

Fig. 1 Support, matching and independent set

For example, in Fig. 1, the vertex subsets $\left\{v_{2}, v_{3}, v_{4}, v_{5}\right\}$ and $\left\{v_{2}, v_{3}, v_{4}, v_{6}\right\}$ of the tree $T_{1}$ are the only independent sets of maximum cardinality. Therefore,

$$
\mathcal{I}(G)=\left\{\left\{v_{2}, v_{3}, v_{4}, v_{5}\right\},\left\{v_{2}, v_{3}, v_{4}, v_{6}\right\},\right.
$$

and $\alpha\left(T_{1}\right)=\left|\left\{v_{2}, v_{3}, v_{4}, v_{5}\right\}\right|=4$.

Definition 2.2 A matching $M$ in $G$ is a set of pairwise non-adjacent edges, that is, no two edges in $M$ share a common vertex. A maximum matching is a matching of largest cardinality in $G$. The matching number of $G$, denoted by $v(G)$, is the size of a set of any maximum matching. $\mathcal{M}(G)$ denotes the set of all maximum matching of $G$. A vertex is saturated by $M$, if it is an endpoint of one of the edges in the matching $M$. Otherwise, the vertex is said non-saturated. Moreover, a matching is said to be perfect if it saturates all vertices of $G$.

In the figures, we use zig zag edges to represent the edges of a matching.

In Fig. 1, tree $T_{1}$ has matching $\left\{\left\{v_{1}, v_{3}\right\},\left\{v_{5}, v_{6}\right\}\right\}$ and tree $T_{2}$ has perfect matching $\left\{\left\{v_{7}, v_{9}\right\},\left\{v_{8}, v_{10}\right\},\left\{v_{11}, v_{14}\right\},\left\{v_{12}, v_{13}\right\}\right\}$. Therefore, $v\left(T_{1}\right)=2$ and $v\left(T_{2}\right)=4$.

The Edmond-Gallai vertices of $G$, denoted by $E G(G)$, is the set of all vertices of $G$ that are non-saturated by some maximum matching $M$ in $G$.

For example, the maximum matchings of $T_{1}$ are

$$
\mathcal{M}\left(T_{1}\right)=\left\{\left\{\left\{v_{1}, v_{2}\right\},\left\{v_{5}, v_{6}\right\}\right\},\left\{\left\{v_{1}, v_{3}\right\},\left\{v_{5}, v_{6}\right\}\right\},\left\{\left\{v_{1}, v_{4}\right\},\left\{v_{5}, v_{6}\right\}\right\}\right\} .
$$

Thus, $E G\left(T_{1}\right)=\left\{v_{2}, v_{3}, v_{4}\right\}$ and $E G\left(T_{2}\right)=\emptyset$.

Definition 2.3 Let $G$ be a graph with $n$ vertices and let $x$ be a vector of $\mathbb{R}^{n}$. The support of $x$ in $G$ is

$$
\operatorname{Supp}_{G}(x)=\left\{v \in V(G): x_{v} \neq 0\right\} .
$$

Let $S$ be a subset of $\mathbb{R}^{n}$. Then the support of $S$ in $G$ is

$$
\operatorname{Supp}_{G}(S)=\bigcup_{x \in S} \operatorname{Supp}_{G}(x) .
$$

As a convention, we use rectangular vertices in figures to represent the vertices of the support. Consider tree $T_{1}$ (Fig. 1) and the set of vectors

$S=\left\{(0,1,0,-1,0,0)^{t},(0,0,1,-1,0,0)^{t}\right\}$. Then $\operatorname{Supp}_{T_{1}}(S)=\left\{v_{2}, v_{3}, v_{4}\right\}$.

The following result shows that to compute the support of an eigenspace of $A(G)$, it is enough to analyse the coordinates of the vectors of a basis of this eigenspace. 
Lemma 2.4 (Jaume and Molina 2018) Let $G$ be a graph, and $\lambda$ an eigenvalue of $A(G)$. Let $\mathcal{B}=\left\{b_{1}, \ldots, b_{k}\right\}$ be a basis of $\varepsilon_{\lambda}$, then $\operatorname{Supp}_{G}\left(\varepsilon_{\lambda}\right)=\operatorname{Supp}_{G}(\mathcal{B})$.

We are interested in the support of the null space of $A(G)$, that is, our focus is $\operatorname{Supp}_{G}(\mathcal{N}(G))$, which, for purposes of notation, is denoted by $\operatorname{Supp}(G)$. In practice, to compute $\operatorname{Supp}(G)$, we will use Lemma 2.4 and not the definition of support. That is, we compute a basis of the null space and consider the entries of the vectors in the basis to obtain the support.

For example, notice that $S=\left\{(0,1,0,-1,0,0)^{t},(0,0,1,-1,0,0)^{t}\right\}$ is a basis of $\mathcal{N}\left(T_{1}\right)$ (Fig. 1); thus,

$$
\operatorname{Supp}\left(T_{1}\right)=\operatorname{Supp}_{T_{1}}(S)=\left\{v_{2}, v_{3}, v_{4}\right\}
$$

Moreover, note that $T_{2}$ (Fig. 1) is non-singular, that is, $\mathcal{N}\left(T_{2}\right)=\{0\}$; therefore, $\operatorname{Supp}\left(T_{2}\right)=\emptyset$.

Theorem 2.5 (Jaume and Molina 2018) Let $T$ be a tree, then Supp $(T)$ is an independent set of $T$.

In Bevis et al. (1995), the authors showed that the rank of tree is twice the matching number. Lemma 2.6 is a corollary of this fact.

Lemma 2.6 $T$ is a nonsingular tree if and only if $T$ has a perfect matching.

As we can see in Fig. 1, tree $T_{1}$ is a singular tree, because it does not have perfect matching. Tree $T_{2}$ is a nonsingular tree, because it has perfect matching.

Our first goal is to characterize singular unicyclic graphs in terms of the support of their pendant trees, which is the subject of next section.

In the next lemma, we show that only the vertices of the support of a tree are not saturated by some maximum matching in this tree.

Lemma 2.7 Let $T$ be a tree, then $E G(T)=\operatorname{Supp}(T)$.

Proof of Lemma 2.7 is in Sect. 4.

\section{Singular unicyclic graphs}

In this section, we characterize singular unicyclic graphs using the support of their pendant trees, which is the statement of Theorem 3.6.

For cycles, the problem of characterizing singular graphs is solved.

Lemma 3.1 (Sookyang et al. 2008) A cycle $C_{n}$ of $n$ vertices is singular if and only if $n$ is divisible by 4 .

Hence, for the remaining of this section, we will consider a unicyclic graph $G \neq C_{n}$. Let $G$ be a unicyclic graph and let $C$ be the unique cycle of $G$. For each vertex $v \in V(C)$, we denote by $G\{v\}$ the induced connected subgraph of $G$ with maximum possible number of vertices, which contains the vertex $v$ and no other vertex of $C . G\{v\}$ is called the pendant tree of $G$ at $v$. Notice that $G$ is obtained by identifying the vertex $v$ of $G\{v\}$ with the vertex $v$ on $C$ for all vertices $v \in C$. In Fig. 2, we have two unicyclic graphs $G$ and $H$ with their pendant trees $G\left\{v_{1}\right\}, G\left\{v_{2}\right\}, G\left\{v_{3}\right\}, H\left\{v_{4}\right\}, H\left\{v_{5}\right\}$ and $H\left\{v_{6}\right\}$, respectively. 


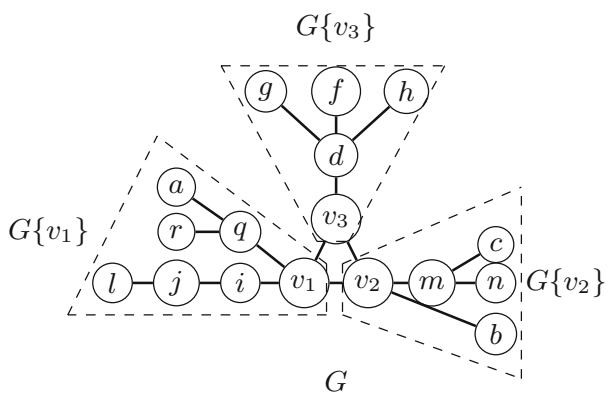

$G$

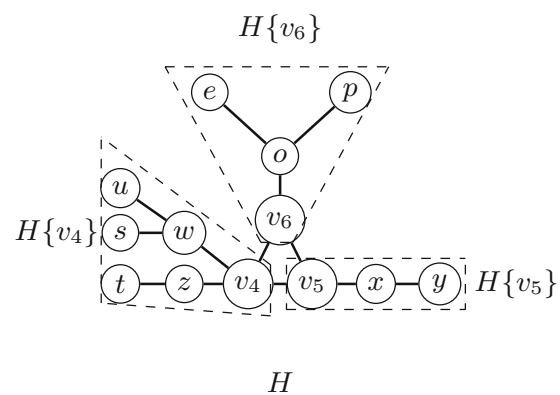

$H$

Fig. 2 Unicyclic graphs of Types I and II and their pendant trees

Definition 3.2 (Gong et al. 2010) For a tree $G\{v\}$ with at least two vertices, vertex $v \in G\{v\}$ is called mismatched in $G\{v\}$ if there exists a maximum matching of $G\{v\}$ that does not saturate $v$; otherwise, $v$ is called matched in $G\{v\}$. If a tree consists of only one vertex it is considered mismatched.

A unicyclic graph $G$ is said to be of Type I if there exists a vertex $v$ on the cycle of $G$ such that $v$ is matched in $G\{v\}$, otherwise, $G$ is said to be of Type II.

To emphasize, a unicyclic graph $G$ is of Type I, if there exists a vertex $v$ of its cycle that is saturated by all maximum matchings of the pendant tree $G\{v\}$. $G$ is of Type II if any vertex $v$ of its cycle is not saturated by some maximum matching of $G\{v\}$.

As an example, consider the unicyclic graph $G$ in Fig. 2. We notice that $G$ is of Type I, because the vertex $v_{1}$ is matched in $G\left\{v_{1}\right\}$. Indeed, the maximum matchings of $G\left\{v_{1}\right\}$ are $\left\{\{a, q\},\left\{v_{1}, i\right\},\{j, l\}\right\}$ and $\left\{\{r, q\},\left\{v_{1}, i\right\},\{j, l\}\right\}$ and both of them saturate $v_{1}$. The unicyclic graph $H$ of Fig. 2 is of Type II, because the pendant trees $H\left\{v_{4}\right\}, H\left\{v_{5}\right\}$ and $H\left\{v_{6}\right\}$ have maximum matchings that do not saturate $v_{4}, v_{5}$ and $v_{6}$, respectively. For example, $\{\{u, w\},\{t, z\}\}$, $\{\{x, y\}\}$ and $\{\{p, o\}\}$ are maximum matchings in $H\left\{v_{4}\right\}, H\left\{v_{5}\right\}$ and $H\left\{v_{6}\right\}$, respectively, that do not saturate $v_{4}, v_{5}$ and $v_{6}$, respectively.

We show next that to verify that a unicyclic graph is Type I or II, it suffices to check whether a vertex $v$ of the cycle is or is not in the support of the pendant tree $G\{v\}$.

Proposition 3.3 A unicyclic graph $G$ is of Type I if and only if there exists at least one pendant tree $G\{v\}$ such that $v \notin \operatorname{Supp}(G\{v\})$.

Proof Since $G$ is of Type I, we know that there exists a vertex $v$ in the cycle of $G$ such that $v$ is always saturated by any maximum matching in $G\{v\}$, that is, $v \notin E G(G\{v\})$, by Lemma 2.7 we have $v \notin \operatorname{Supp}(G\{v\})$.

Immediately, we obtain the dual result.

Corollary 3.4 A unicyclic graph $G$ is of Type II if and only if every pendant tree $G\{v\}$ is such that $v \in \operatorname{Supp}(G\{v\})$.

The following result computes the nullity of a unicyclic graph from the nullity of its pendant trees.

Lemma 3.5 (Gong et al. 2010) Let $G$ be a unicyclic graph and let $C$ be its cycle. If $G$ is of Type I and $v \in V(C)$ be matched in $G\{v\}$, then

$$
\eta(G)=\eta(G\{v\})+\eta(G-G\{v\}) .
$$


If $G$ is of Type II, then

$$
\eta(G)=\eta(G-C)+\eta(C) .
$$

We now obtain a characterization of singular unicyclic graphs using the support of their pendant trees.

Theorem 3.6 Let $G$ be a unicyclic graph and let $C$ be the cycle of $G . G$ is singular if and only if one of the following happens:

(i) There is a pendant tree $G\{v\}$, with $v \notin \operatorname{Supp}(G\{v\})$ and either $G\{v\}$ does not have perfect matching or $G-G\{v\}$ does not have perfect matching.

(ii) Every pendant tree $G\{v\}$ is such that $v \in \operatorname{Supp}(G\{v\})$ and either one of the trees that compose the forest $G-C$ does not have perfect matching or the cycle $C$ has length equal to a multiple of 4 .

Proof (i) As there is a pendant tree $G\{v\}$ such that $v \notin \operatorname{Supp}(G\{v\})$, we conclude by Proposition 3.3 that $G$ is of Type I. Moreover, by Lemma 3.5, we conclude that $G$ will be singular if and only if $G\{v\}$ or $G-G\{v\}$ has nonzero nullity, and by Lemma 2.6, we know that this only happens if $G\{v\}$ does not have perfect matching or $G-G\{v\}$ does not have perfect matching.

(ii) Since every pendant tree $G\{v\}$ is such that $v \in \operatorname{Supp}(G\{v\})$, we conclude by Corollary 3.4 that $G$ is of Type II. Then by Lemma 3.5, we know that $G$ will be singular if and only if $G-C$ or $C$ has nonzero nullity, and by Lemmas 2.6 and 3.1, it happens if and only if at least one of the trees composing the forest $G-C$ does not have perfect matching or the cycle $C$ has length equal to a multiple of 4 .

\section{Independence number of unicyclic graphs}

In this section, we obtain closed formulas for the independence number of a unicyclic graph $G$. This formula depends on the number of vertices of the support and also on the amount of $N$-vertices of subtrees of this unicyclic graph $G$. To understand our result, we start by presenting the null decomposition of trees, given in Jaume and Molina (2018).

Definition 4.1 Let $T$ be a tree. The $S$-forest of $T$, denoted by $\mathcal{F}_{S}(T)$, is defined as the subgraph induced by the closed neighborhood of $\operatorname{Supp}(T)$ in $T$ :

$$
\mathcal{F}_{S}(T)=T[N[\operatorname{Supp}(T)]] .
$$

The $N$-forest of $T$, denoted by $\mathcal{F}_{N}(T)$, is defined as the remaining graph:

$$
\mathcal{F}_{N}(T)=T-\mathcal{F}_{S}(T) .
$$

The null decomposition of $T$ is the pair $\left(\mathcal{F}_{S}(T), \mathcal{F}_{N}(T)\right)$.

$V\left(\mathcal{F}_{N}(T)\right)$ is called the set of $N$-vertices of $T$.

We represent star vertices in the figures as the $N$-vertices. As an example, the support of the tree $T$ in Fig. 3 is

$$
\operatorname{Supp}(T)=\left\{v_{2}, v_{3}, v_{6}, v_{7}, v_{8}, v_{10}, v_{11}, v_{12}, v_{19}, v_{21}, v_{22}\right\} .
$$




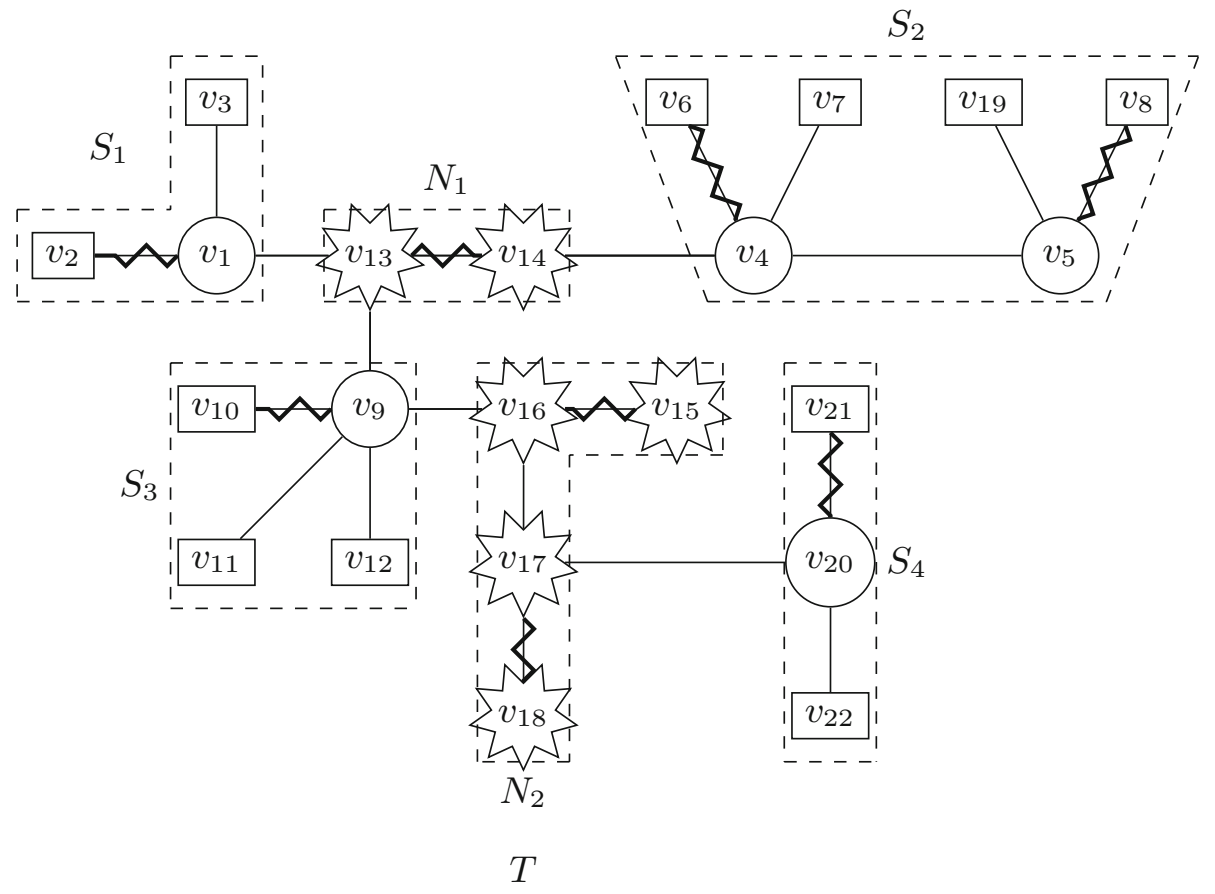

Fig. 3 Null decomposition of the tree $T$

The $S$-forest of $T$ generated by the closed neighborhood of the support consists of

$$
\begin{aligned}
\mathcal{F}_{S}(T) & =T[N[\operatorname{Supp}(T)]]=T\left[N\left[\left\{v_{2}, v_{3}, v_{6}, v_{7}, v_{8}, v_{10}, v_{11}, v_{12}, v_{19}, v_{21}, v_{22}\right\}\right]\right] \\
& =T\left[\left\{v_{1}, v_{2}, v_{3}, v_{4}, v_{5}, v_{6}, v_{7}, v_{8}, v_{9}, v_{10}, v_{11}, v_{12}, v_{19}, v_{20}, v_{21}, v_{22}\right\}\right] \\
& =S_{1} \cup S_{2} \cup S_{3} \cup S_{4} .
\end{aligned}
$$

The $N$-forest of $T$ consists of

$$
\mathcal{F}_{N}(T)=T-\mathcal{F}_{S}(T)=T\left[\left\{v_{13}, v_{14}, v_{15}, v_{16}, v_{17}, v_{18}\right\}\right]=N_{1} \cup N_{2} .
$$

Figure 3 illustrates the null decomposition of the tree $T$.

Definition 4.2 The core of $G$, denoted by $\operatorname{Core}(G)$, is defined to be the set of all the neighbours of some supported vertex of $G$ :

$$
\operatorname{Core}(G)=\bigcup_{v \in \operatorname{Supp}(G)} N(v) .
$$

For example, the core of tree $T$ (Fig. 3) is

$$
\operatorname{Core}(T)=\left\{v_{1}, v_{4}, v_{5}, v_{9}, v_{20}\right\} .
$$

The next lemma gives closed formulas for the independence and matching numbers of trees and it is crucial to prove our main results. 
Lemma 4.3 (Jaume and Molina 2018) Let $T$ be a tree. Then

$$
\begin{gathered}
v(T)=|\operatorname{Core}(T)|+\frac{\left|V\left(\mathcal{F}_{N}(T)\right)\right|}{2} \\
\alpha(T)=|\operatorname{Supp}(T)|+\frac{\left|V\left(\mathcal{F}_{N}(T)\right)\right|}{2} .
\end{gathered}
$$

We use Lemma 4.4 to show Lemma 2.7.

Lemma 4.4 Let $T$ be a tree. Consider $\mathcal{F}_{S}(T)=\bigcup_{i=1}^{k} S_{i}$ and $\mathcal{F}_{N}(T)=\bigcup_{j=1}^{t} N_{j}$, where for all $i \in\{1, \ldots, k\}$ and $j \in\{1, \ldots, t\}$ we have that $S_{i}$ and $N_{j}$ are connected components of $\mathcal{F}_{S}(T)$ and $\mathcal{F}_{N}(T)$, respectively. If $M \in \mathcal{M}(T)$, then $M \cap E\left(S_{i}\right) \in \mathcal{M}\left(S_{i}\right)$ and $M \cap E\left(N_{j}\right) \in$ $\mathcal{M}\left(N_{j}\right)$, where $i \in\{1, \ldots, k\}$ and $j \in\{1, \ldots, t\}$.

Proof Suppose there exists $M \in \mathcal{M}(T)$ such that $M \cap E\left(S_{r}\right) \notin \mathcal{M}\left(S_{r}\right)$ or $M \cap E\left(N_{s}\right) \notin$ $\mathcal{M}\left(N_{s}\right)$, for some $r \in\{1, \ldots, k\}$ or $s \in\{1, \ldots, t\}$, that is, $\left|M \cap E\left(S_{r}\right)\right|<v\left(S_{r}\right)$ or $\left|M \cap E\left(N_{s}\right)\right|<v\left(N_{s}\right)$. By Corollary 4.14 of Jaume and Molina (2018), we obtain

$$
M \cap\left(E(T)-\left(E\left(\mathcal{F}_{S}(T)\right) \cup E\left(\mathcal{F}_{N}(T)\right)\right)\right)=\emptyset .
$$

Then $M=\left(M \cap E\left(\mathcal{F}_{S}(T)\right)\right) \cup\left(M \cap E\left(\mathcal{F}_{N}(T)\right)\right)$. By Corollary 3.8 and Corollary 4.6 of Jaume and Molina (2018), we obtain $v\left(S_{i}\right)=\left|\operatorname{Core}\left(S_{i}\right)\right|$, for all $i \in\{1, \ldots, k\}$ and $\operatorname{Core}(T)=\bigcup_{i=1}^{k} \operatorname{Core}\left(S_{i}\right)$, respectively. Moreover, by Theorem 4.13 of Jaume and Molina (2018), we have that $N_{j}$ is nonsingular tree, for all $j \in\{1, \ldots, t\}$. Therefore, by Lemma 2.6, we conclude that $N_{j}$ has perfect matching and $v\left(N_{j}\right)=\frac{\left|V\left(N_{j}\right)\right|}{2}$. Thus, we have that

$$
\begin{aligned}
v(T) & =|M|=\left|M \cap E\left(\mathcal{F}_{S}(T)\right)\right|+\left|M \cap E\left(\mathcal{F}_{N}(T)\right)\right| \\
& =\sum_{i=1}^{k}\left|M \cap E\left(S_{i}\right)\right|+\sum_{j=1}^{t}\left|M \cap E\left(N_{j}\right)\right| \\
& <\sum_{i=1}^{k} v\left(S_{i}\right)+\sum_{j=1}^{t} v\left(N_{j}\right)=|\operatorname{Core}(T)|+\frac{\left|V\left(\mathcal{F}_{N}(T)\right)\right|}{2},
\end{aligned}
$$

which is a contradiction, because by Lemma 4.3, we have that $v(T)=|\operatorname{Core}(T)|+\frac{\left|V\left(\mathcal{F}_{N}(T)\right)\right|}{2}$.

Proof of Lemma 2.7 Let $T$ be a tree. Consider $\mathcal{F}_{S}(T)=\bigcup_{i=1}^{k} S_{i}$ and $\mathcal{F}_{N}(T)=\bigcup_{j=1}^{t} N_{j}$, where for all $i \in\{1, \ldots, k\}$ and $j \in\{1, \ldots, t\}$, we have that $S_{i}$ and $N_{j}$ are connected components of $\mathcal{F}_{S}(T)$ and $\mathcal{F}_{N}(T)$, respectively. Given $v \in \operatorname{Supp}(T)$. We will obtain $M \in$ $\mathcal{M}(T)$ such that $M$ does not saturate $v$. By Corollary 4.6 of Jaume and Molina (2018), we have that $\operatorname{Supp}(T)=\bigcup_{i=1}^{k} \operatorname{Supp}\left(S_{i}\right)$ and $\operatorname{Core}(T)=\bigcup_{i=1}^{k} \operatorname{Core}\left(S_{i}\right)$. Thus, $v \in \operatorname{Supp}\left(S_{r}\right)$, for some $r \in\{1, \ldots, k\}$. By Theorem 4.13 of Jaume and Molina (2018), we have that there exists $M_{r} \in \mathcal{M}\left(S_{r}\right)$ such that $v$ is not saturated by $M_{r}$. Consider $M_{i} \in \mathcal{M}\left(S_{i}\right)$ and $M_{j}^{\prime} \in \mathcal{M}\left(N_{j}\right)$, where $i \in\{1, \ldots, k\}-\{r\}$ and $j \in\{1, \ldots, t\}$. Define $M=\left(\bigcup_{i=1}^{k} M_{i}\right) \cup\left(\bigcup_{j=1}^{t} M_{j}^{\prime}\right)$. Note that $M$ is a matching in $T$ and $|M|=v(T)$, that is, $M \in \mathcal{M}(T)$. Moreover, $v$ is not saturated by $M$. Therefore, $v \in E G(T)$.

Now, given $v \in E G(T)$. We will show that $v \in \operatorname{Supp}(T)$. Since $v \in E G(T)$, then there exists $M \in \mathcal{M}(T)$ such that $v$ is not saturated by $M$. Note that by Theorem 4.13 of Jaume and Molina (2018), we have that $N_{j}$ is nonsingular tree, for all $j \in\{1, \ldots, t\}$. Thus, by Lemma 2.6, we conclude that $N_{j}$ has perfect matching. Moreover, by Lemma 4.4, we obtain 
$M \cap E\left(N_{j}\right) \in \mathcal{M}\left(N_{j}\right)$, where $j \in\{1, \ldots, t\}$, that is, $M \cap E\left(N_{j}\right)$ is a perfect matching in $N_{j}$. Hence, $v \notin \bigcup_{j=1}^{t} V\left(N_{j}\right)$. Then $v \in V\left(S_{r}\right)$, for some $r \in\{1, \ldots, k\}$. Since $v$ is not saturated by $M$, then $v$ is not saturated by $M \cap E\left(S_{r}\right)$. Notice that $M \cap E\left(S_{r}\right) \in \mathcal{M}\left(S_{r}\right)$, by Lemma 4.4. Thus, by Theorem 4.13 of Jaume and Molina (2018), we conclude that $v \in \operatorname{Supp}\left(S_{r}\right)$. By Corollary 4.6 of Jaume and Molina (2018), we have that $\operatorname{Supp}(T)=\bigcup_{i=1}^{k} \operatorname{Supp}\left(S_{i}\right)$. Therefore, $v \in \operatorname{Supp}(T)$.

Our next result tells us that given any vertex in a nonsingular tree, there will always be at least one maximum independent set that does not contain this vertex and another maximum independent set that contains this vertex.

Proposition 4.5 Let $T$ be a nonsingular tree and $v \in V(T)$. Then there exist $I_{1}, I_{2} \in \mathcal{I}(T)$ such that $v \in I_{1}$ and $v \notin I_{2}$.

Proof Since $T$ is a tree, we have that $T$ is a bipartite graph. Then there exist two disjoint subsets $B_{1}$ and $B_{2}$ of $V(T)$ such that $V(T)=B_{1} \cup B_{2}$ and for all $\{a, b\} \in E(T)$, we have $\{a, b\} \cap B_{1} \neq \varnothing$ and $\{a, b\} \cap B_{2} \neq \varnothing$. As $T$ is a nonsingular tree, it has perfect matching $M$, by Lemma 2.6. As $\alpha(T)=v(T)=|M|=\frac{|V(T)|}{2}$ and for all $\{a, b\} \in M$, we have $\{a, b\} \cap B_{1} \neq \emptyset$ and $\{a, b\} \cap B_{2} \neq \emptyset$, then $\left|B_{1}\right|=\left|B_{2}\right|=\frac{|V(T)|}{2}$. That is, $B_{1}, B_{2} \in \mathcal{I}(T)$. Therefore, given a $v \in V(T)$ we have $v \in B_{1}$ and $v \notin B_{2}$ or $v \in B_{2}$ and $v \notin B_{1}$.

Lemma 4.6 If $T$ is a tree and $v \in V\left(\mathcal{F}_{N}(T)\right)$, then there exist $I_{1}, I_{2} \in \mathcal{I}(T)$ such that $v \in I_{1}$ and $v \notin I_{2}$.

Proof The null decomposition, in general, divides a tree $T$ into two forests (one of them may be empty), a forest formed by singular trees, denoted by $\mathcal{F}_{S}(T)$, and other formed by non-singular trees, denoted by $\mathcal{F}_{N}(T)$ (see Theorem 4.5 and Theorem 4.13 of Jaume and Molina 2018).

Moreover, we have $\operatorname{Supp}(T)=\bigcup_{S \in \mathcal{F}_{S}(T)} \operatorname{Supp}(S)$ and $V\left(\mathcal{F}_{N}(T)\right)=\bigcup_{N \in \mathcal{F}_{N}(T)} V(N)$. Thus, if $v \in V\left(\mathcal{F}_{N}(T)\right)$, we have $v \in V\left(N_{1}\right)$ for some $N_{1} \in \mathcal{F}_{N}(T)$. As $N_{1}$ is non-singular using Proposition 4.5, we obtain $I_{N_{1}}, J_{N_{1}} \in \mathcal{I}\left(N_{1}\right)$ such that $v \in J_{N_{1}}$ and $v \notin I_{N_{1}}$.

Let

$$
\begin{aligned}
& I_{1}=\operatorname{Supp}(T) \cup I_{N_{1}} \cup\left(\bigcup_{N \in \mathcal{F}_{N}(T) \text { and } N \neq N_{1}} I_{N}\right) \text { and } \\
& I_{2}=\operatorname{Supp}(T) \cup J_{N_{1}} \cup\left(\bigcup_{N \in \mathcal{F}_{N}(T) \text { and } N \neq N_{1}} J_{N}\right) .
\end{aligned}
$$

We observe that $I_{1}$ and $I_{2}$ are independent sets because $\operatorname{Supp}(T)$ is an independent set of $T$ and $N(\operatorname{Supp}(T))=\operatorname{Core}(T)$, then $N(\operatorname{Supp}(T)) \cap\left(\bigcup_{N \in \mathcal{F}_{N}(T)} I_{N}\right)=\emptyset$. Notice that $\left|I_{1}\right|=\left|I_{2}\right|=|\operatorname{Supp}(T)|+\frac{\left|V\left(\mathcal{F}_{N}(T)\right)\right|}{2}$, then $I_{1}, I_{2} \in \mathcal{I}(T)$ by Lemma 4.3. Moreover, we have $v \notin I_{1}$ and $v \in I_{2}$.

Lemma 4.7 Let $T$ be a tree and I an independent set of $T$. If $c_{i} \in \operatorname{Core}(T) \cap I$, then $I \notin \mathcal{I}(T)$.

Proof We notice that, in general, $I=\left\{s_{1}, s_{2}, \ldots, s_{j}\right\} \cup\left\{c_{1}, c_{2}, \ldots, c_{t}\right\} \cup\left\{n_{1}, n_{2}, \ldots, n_{r}\right\}$, where $\left\{s_{1}, s_{2}, \ldots, s_{j}\right\} \subseteq \operatorname{Supp}(T),\left\{c_{1}, c_{2}, \ldots, c_{t}\right\} \subseteq \operatorname{Core}(T)$ and $\left\{n_{1}, n_{2}, \ldots, n_{r}\right\} \subseteq$ 
$V\left(\mathcal{F}_{N}(T)\right.$ ) (possibly we can have $\left\{n_{1}, n_{2}, \ldots, n_{r}\right\}=\emptyset$ ). By Lemma 3.5 of Jaume and Molina (2018), we have

$$
\left|N\left(\left\{c_{1}, c_{2}, \ldots, c_{t}\right\}\right) \cap \operatorname{Supp}(T)\right|>\left|\left\{c_{1}, c_{2}, \ldots, c_{t}\right\}\right| .
$$

Note that $J=\left\{s_{1}, s_{2}, \ldots, s_{j}\right\} \cup\left(N\left(\left\{c_{1}, c_{2}, \ldots, c_{t}\right\}\right) \cap \operatorname{Supp}(T)\right) \cup\left\{n_{1}, n_{2}, \ldots, n_{r}\right\}$ is an independent set of $T$. Indeed, $N(\operatorname{Supp}(T)) \cap\left\{n_{1}, n_{2}, \ldots, n_{r}\right\}=\emptyset$ and $\left\{s_{1}, s_{2}, \ldots, s_{j}\right\}$ and $N\left(\left\{c_{1}, c_{2}, \ldots, c_{t}\right\}\right) \cap \operatorname{Supp}(T)$ are independent sets, because $\left\{s_{1}, s_{2}, \ldots, s_{j}\right\} \subseteq \operatorname{Supp}(T)$, $N\left(\left\{c_{1}, c_{2}, \ldots, c_{t}\right\}\right) \cap \operatorname{Supp}(T) \subseteq \operatorname{Supp}(T)$ and $\operatorname{Supp}(T)$ is an independent set by Theorem 2.5. Moreover, note that $\left\{s_{1}, s_{2}, \ldots, s_{j}\right\} \cap\left(N\left(\left\{c_{1}, c_{2}, \ldots, c_{t}\right\}\right) \cap \operatorname{Supp}(T)\right)=\emptyset$ otherwise $I$ would not be an independent set, then $|J|>|I|$, therefore, $I \notin \mathcal{I}(T)$.

Theorem 4.8 is one of the main results of this section. It gives a closed formula for the independence number of unicyclic graphs $G$ of Type I. This formula depends on the support and $N$-vertices of subtrees. It means that using this formula, we can compute the independence number of unicyclic graphs of Type I using linear algebra.

Theorem 4.8 If $G$ is a unicyclic graph of Type I and $G\{v\}$ its pendant tree such that $v \notin$ $\operatorname{Supp}(G\{v\})$, then

$$
\alpha(G)=|\operatorname{Supp}(G\{v\})|+|\operatorname{Supp}(G-G\{v\})|+\frac{\left|V\left(\mathcal{F}_{N}(G\{v\})\right)\right|+\left|V\left(\mathcal{F}_{N}(G-G\{v\})\right)\right|}{2} .
$$

Proof Note that there is an independent set $I_{1} \in \mathcal{I}(G\{v\})$ such that $v \notin I_{1}$. Indeed, if $v \in V\left(\mathcal{F}_{N}(G\{v\})\right) \cup \operatorname{Core}(G\{v\})$, then by Lemmas 4.6 and 4.7 there is a $I_{1} \in \mathcal{I}(G\{v\})$ such that $v \notin I_{1}$. Let $I_{2} \in \mathcal{I}(G-G\{v\})$. Let $u, w \in N(v) \cap V(G-G\{v\})$. We will prove that $I_{1} \cup I_{2} \in \mathcal{I}(G)$.

We notice that $I_{1} \cup I_{2}$ is an independent set in $G$. To see that, we observe that the vertices of $I_{1}$ are not connected to each other, because $I_{1}$ is an independent set. Similarly, we conclude the same for $I_{2}$. Moreover, the only adjacencies between $G\{v\}$ and $G-G\{v\}$ occur between vertices $v$ and $u$ and vertices $v$ and $w$. Since $v \notin I_{1}$, there is no possibility of adjacency between vertices of $I_{1}$ and vertices of $I_{2}$. Suppose that $I_{1} \cup I_{2} \notin \mathcal{I}(G)$, that is, there exists an independent set $J$ in $G$ such that $|J|>\left|I_{1} \cup I_{2}\right|$. As $V(G)=V(G\{v\}) \cup V(G-G\{v\})$, there is a $J_{1} \subseteq V(G\{v\})$ and $J_{2} \subseteq V(G-G\{v\})$ such that $J=J_{1} \cup J_{2}$. We have that

$$
\left|I_{1}\right|+\left|I_{2}\right|=\left|I_{1} \cup I_{2}\right|<|J|=\left|J_{1}\right|+\left|J_{2}\right| .
$$

In this case, we see that $J_{1}$ and $J_{2}$ are independent sets in $G\{v\}$ and $G-G\{v\}$, respectively. Thus, we have $\left|J_{1}\right| \leq\left|I_{1}\right|$ and $\left|J_{2}\right| \leq\left|I_{2}\right|$, because $I_{1} \in \mathcal{I}(G\{v\})$ and $I_{2} \in \mathcal{I}(G-G\{v\})$. Therefore, $\left|J_{1}\right|+\left|J_{2}\right| \leq\left|I_{1}\right|+\left|I_{2}\right|$, which is a contradiction by (1). Hence, $I_{1} \cup I_{2} \in \mathcal{I}(G)$. By Lemma 4.3, we have

$$
\begin{gathered}
\alpha(G\{v\})=|\operatorname{Supp}(G\{v\})|+\frac{\left|V\left(\mathcal{F}_{N}(G\{v\})\right)\right|}{2} \text { and } \\
\alpha(G-G\{v\})=|\operatorname{Supp}(G-G\{v\})|+\frac{\left|V\left(\mathcal{F}_{N}(G-G\{v\})\right)\right|}{2} .
\end{gathered}
$$

Therefore, the independence number of $G$ is given by

$$
\begin{aligned}
\alpha(G) & =\left|I_{1}\right|+\left|I_{2}\right|=\alpha(G\{v\})+\alpha(G-G\{v\}) \\
& =|\operatorname{Supp}(G\{v\})|+\frac{\left|V\left(\mathcal{F}_{N}(G\{v\})\right)\right|}{2}+|\operatorname{Supp}(G-G\{v\})|+\frac{\left|V\left(\mathcal{F}_{N}(G-G\{v\})\right)\right|}{2} .
\end{aligned}
$$




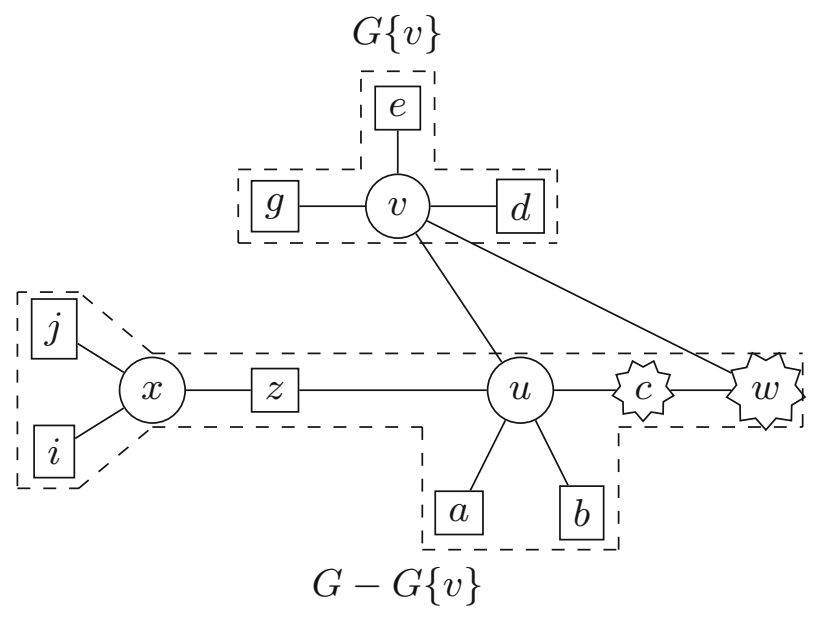

Fig. 4 Unicyclic graph $G$ and its subtrees

The following example is an application of Theorem 4.8. Consider $G$ the unicyclic graph of Fig. 4. We observe that $G$ is of Type I. Indeed, $v \notin \operatorname{Supp}(G\{v\})=\{g, e, d\}$, then by Proposition 3.3, we have that $G$ is a unicyclic graph of Type I. Moreover, $\operatorname{Supp}(G-G\{v\})=$ $\{a, b, i, j, z\}, V\left(\mathcal{F}_{N}(G\{v\})\right)=\emptyset$ and $V\left(\mathcal{F}_{N}(G-G\{v\})=\{w, c\}\right.$.

Therefore, by Theorem 4.8, we have that the independence number of $G$ is given by

$$
\begin{aligned}
\alpha(G) & =|\operatorname{Supp}(G\{v\})|+|\operatorname{Supp}(G-G\{v\})|+\frac{\left|V\left(\mathcal{F}_{N}(G\{v\})\right)\right|+\left|V\left(\mathcal{F}_{N}(G-G\{v\})\right)\right|}{2} \\
& =3+5+\frac{2}{2}=9 .
\end{aligned}
$$

We observe that $J=\{a, b, c, d, e, g, i, j, c\}$ is a maximum independent set of $G$ and $|J|=9$.

Lemma 4.9 Let $G$ be a unicyclic graph and $C$ its cycle. Let $G\{v\}$ be a pendant tree such that $v \in \operatorname{Supp}(G\{v\})$. If $u \in N(v) \cap V(G\{v\})$, then $u \notin \operatorname{Supp}(G-C)$.

Proof Let $G\{v\}-v=\bigcup_{i=1}^{k} T_{i}$, where $T_{i}$ is a connected component of $G\{v\}-v$. Let $M_{i} \in \mathcal{M}\left(T_{i}\right)$. As $v \in \operatorname{Supp}(G\{v\})$ there is a $M \in \mathcal{M}(G\{v\})$ such that $M$ does not saturate $v$ by Lemma 2.7. We observe that $u \in N(v) \cap V(G\{v\})$, then $u \notin \operatorname{Supp}(G\{v\})$, because $\operatorname{Supp}(G\{v\})$ is an independent set of $G\{v\}$ by Theorem 2.5. Then $M \in \mathcal{M}(G\{v\}-v)$ and $v(G\{v\})=v(G\{v\}-v)$. Suppose $u \in \operatorname{Supp}(G\{v\}-v)=\bigcup_{i=1}^{k} \operatorname{Supp}\left(T_{i}\right)$, that is, there is a $i$ such that $u \in \operatorname{Supp}\left(T_{i}\right)$, then by Lemma 2.7 in $T_{i}$ we obtain a $M_{i} \in \mathcal{M}\left(T_{i}\right)$ does not saturate $u$. Note that $\bigcup_{i=1}^{k} M_{i} \in \mathcal{M}(G\{v\}-v)$ and $\bigcup_{i=1}^{k} M_{i}$ does not saturate $u$. Then $\bigcup_{i=1}^{k} M_{i} \in \mathcal{M}(G\{v\})$, because $v(G\{v\})=v(G\{v\}-v)$. Which is a contradiction, because $\bigcup_{i=1}^{k} M_{i}$ does not saturate $u$ and all maximum matching in $G\{v\}$ saturates $u$ because $u \notin \operatorname{Supp}(G\{v\})$. Therefore, $u \notin \operatorname{Supp}(G\{v\}-v)$. Since the connected components of $G\{v\}-v$ are connected components of $G-C$, we have $u \notin \operatorname{Supp}(G-C)$.

Theorem 4.10 is a similar result for unicyclic graphs of Type II and gives a closed formula for the independence number of unicyclic graphs of Type II. 
Theorem 4.10 Let $G$ be a unicyclic graph and $C$ its cycle. Let $G-C=\bigcup_{i=1}^{k} T_{i}$, where $T_{i}$ is a connected component of $G-C$. If $G$ is a unicyclic graph of Type II, then

$$
\alpha(G)=\left\lfloor\frac{|V(C)|}{2}\right\rfloor+\sum_{i=1}^{k}\left|\operatorname{Supp}\left(T_{i}\right)\right|+\frac{\left|V\left(\mathcal{F}_{N}\left(T_{i}\right)\right)\right|}{2} .
$$

Proof Let $v_{i} \in V(C)$ and $u_{i} \in T_{i}$ such that $u_{i} \in N\left(v_{i}\right)$. By Lemma 4.9, we have $u_{i} \notin$ $\operatorname{Supp}\left(T_{i}\right)$. As $u_{i} \in V\left(\mathcal{F}_{N}\left(T_{i}\right)\right) \cup \operatorname{Core}\left(T_{i}\right)$ then, by Lemmas 4.6 and 4.7, we have a $J_{i} \in \mathcal{I}\left(T_{i}\right)$ such that $u_{i} \notin J_{i}$. Consider $J_{c} \in \mathcal{I}(C)$ and define $I=J_{c} \cup\left(\bigcup_{i=1}^{k} J_{i}\right)$. We will show that $I \in \mathcal{I}(G)$.

First, we notice that $I$ is an independent set in $G$. Indeed, for all $i$, we observe that the vertices of $J_{i}$ are nonadjacent to each other, because $J_{i}$ is an independent set. Similarly, we conclude the same for $J_{c}$. Moreover, there is no chance that a vertex of $J_{i}$ is adjacent to a vertex $J_{\ell}$, with $i \neq \ell$, since the vertices of the trees $T_{i}$ and $T_{\ell}$ are not adjacent to each other. Now, we show that vertices of $J_{i}$ are not connected to vertices of $J_{c}$. To see that, we observe that the only adjacency that exists between $T_{i}$ and $C$ is the adjacency between vertex $u_{i}$ and $v_{i}$, but since $u_{i} \notin J_{i}$, there is no possibility of adjacency between vertices of $J_{i}$ and $J_{c}$.

Suppose now $I \notin \mathcal{I}(G)$, that is, there is an independent set $F$ in $G$ such that $|F|>|I|$. As $V(G)=V(C) \cup\left(\bigcup_{i=1}^{k} V\left(T_{i}\right)\right)$, we see that there exist $F_{c} \subseteq V(C)$ and $F_{i} \subseteq V\left(T_{i}\right)$ such that $F=F_{c} \cup\left(\bigcup_{i=1}^{k} F_{i}\right)$. Thus, we have

$$
\left|F_{c}\right|+\sum_{i=1}^{k}\left|F_{i}\right|=|F|>|I|=\left|J_{c}\right|+\sum_{i=1}^{k}\left|J_{i}\right| .
$$

As $F_{c}$ and $F_{i}$ are independent sets of $C$ and $T_{i}$, respectively, we have $\left|F_{c}\right| \leq\left|J_{c}\right|$ and $\left|F_{i}\right| \leq$ $\left|J_{i}\right|$, because $J_{c} \in \mathcal{I}(C)$ and $J_{i} \in \mathcal{I}\left(T_{i}\right)$. Thus, we have $\left|F_{c}\right|+\sum_{i=1}^{k}\left|F_{i}\right| \leq\left|J_{c}\right|+\sum_{i=1}^{k}\left|J_{i}\right|$, which is a contradiction by (2). Therefore, $I \in \mathcal{I}(G)$. We observe that $\alpha(C)=\left\lfloor\frac{|V(C)|}{2}\right\rfloor$ and, by Lemma 4.3, we have, for all $i$,

$$
\alpha\left(T_{i}\right)=\left|\operatorname{Supp}\left(T_{i}\right)\right|+\frac{\left|V\left(\mathcal{F}_{N}\left(T_{i}\right)\right)\right|}{2} .
$$

Therefore, the independence number of $G$ is given by

$$
\begin{aligned}
\alpha(G) & =\left|J_{C}\right|+\sum_{i=1}^{k}\left|J_{i}\right|=\alpha(C)+\sum_{i=1}^{k} \alpha\left(T_{i}\right) \\
& =\left\lfloor\frac{|V(C)|}{2}\right\rfloor+\sum_{i=1}^{k}\left|\operatorname{Supp}\left(T_{i}\right)\right|+\frac{\left|V\left(\mathcal{F}_{N}\left(T_{i}\right)\right)\right|}{2} .
\end{aligned}
$$

As an example, consider $G$ the unicyclic graph of Fig. 5. We first notice that $G$ is a unicyclic graph of Type II, because $v \in \operatorname{Supp}(G\{v\})=\{n, p, v\}, w \in \operatorname{Supp}(G\{w\})=$ $\{e, g, h, i, w, d, f\}$ and $u \in \operatorname{Supp}(G\{u\})=\{u, \ell\}$. Then by Corollary 3.4, we obtain $G$ is a unicyclic graph of Type II. Moreover, we have $G-C_{3}=\bigcup_{i=1}^{5} T_{i}$, where $T_{1}=G[\{b, g, h, i\}]$, $T_{2}=G[\{a, e\}], T_{3}=G[\{c, f, d\}], T_{4}=G[\{j, \ell\}]$ and $T_{5}=G[\{m, n, o, p\}]$ (see Fig. 5).

Since $T_{2}, T_{4}$ and $T_{5}$ have perfect matching, so they are non-singular and have empty support. $T_{1}$ and $T_{3}$ do not have perfect matching, so they are singular and computing their supports we obtain Table 1. 


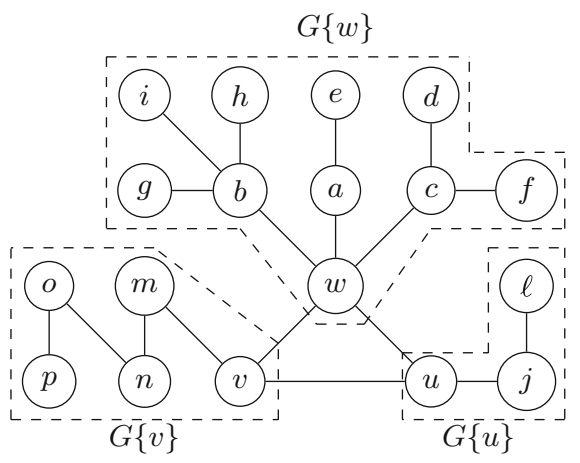

$G$

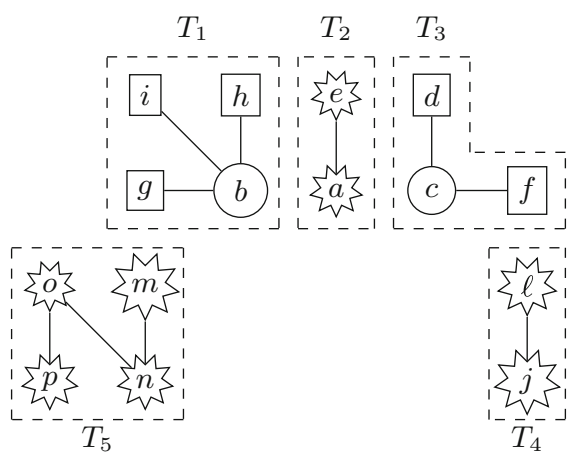

$G-C_{3}$

Fig. 5 Unicyclic graph of Type II and the support of its subtrees

Table 1 Support and $N$-vertices of the subtrees $T_{1}, T_{2}, T_{3}, T_{4}$ and $T_{5}$

\begin{tabular}{lll}
\hline$G-C_{3}$ & Support & $N$-vertices \\
\hline$T_{1}$ & $\operatorname{Supp}\left(T_{1}\right)=\{g, h, i\}$ & $V\left(\mathcal{F}_{N}\left(T_{1}\right)\right)=\emptyset$ \\
$T_{2}$ & $\operatorname{Supp}\left(T_{2}\right)=\emptyset$ & $V\left(\mathcal{F}_{N}\left(T_{2}\right)\right)=\{a, e\}$ \\
$T_{3}$ & $\operatorname{Supp}\left(T_{3}\right)=\{d, f\}$ & $V\left(\mathcal{F}_{N}\left(T_{3}\right)\right)=\emptyset$ \\
$T_{4}$ & $\operatorname{Supp}\left(T_{4}\right)=\emptyset$ & $V\left(\mathcal{F}_{N}\left(T_{4}\right)\right)=\{j, \ell\}$ \\
$T_{5}$ & $\operatorname{Supp}\left(T_{5}\right)=\emptyset$ & $V\left(\mathcal{F}_{N}\left(T_{5}\right)\right)=\{m, n, o, p\}$
\end{tabular}

Therefore, by Theorem 4.10, we have that the independence number of $G$ is given by

$$
\begin{aligned}
\alpha(G) & =\left\lfloor\frac{|V(C)|}{2}\right\rfloor+\sum_{i=1}^{k}\left|\operatorname{Supp}\left(T_{i}\right)\right|+\frac{\left|V\left(\mathcal{F}_{N}\left(T_{i}\right)\right)\right|}{2} \\
& =\left\lfloor\frac{3}{2}\right\rfloor+3+\frac{2}{2}+2+\frac{4}{2}+\frac{2}{2}=10 .
\end{aligned}
$$

We observe that $I=\{g, h, i, e, d, f, p, n, \ell, v\}$ is a maximum independent set of $G$ and $|I|=10$.

\section{Matching number of unicyclic graphs}

In this section, we obtain closed formulas for the matching number of unicyclic graphs.

Definition 5.1 Let $M$ be a matching in the graph $G$. An $M$-alternating path is a path that alternates edges in $M$ and edges that are not in $M$. An $M$-augmenting path is an $M$-alternating path, if it begins and ends at vertices non saturated by $M$.

Consider the matching $M=\{\{d, c\},\{a, b\},\{v, w\}\}$ in graph $G$ of Fig. 6. As the path $P_{1}=(e, d, c, b, a)$ is an $M$-alternating path because its edges alternate outside and within the matching $M$. Now the path $P_{2}=(u, v, w, o)$ is $M$-augmenting, because it is an $M$ alternating path and starts and ends at vertices non-saturated by $M$ (vertices u and o). 


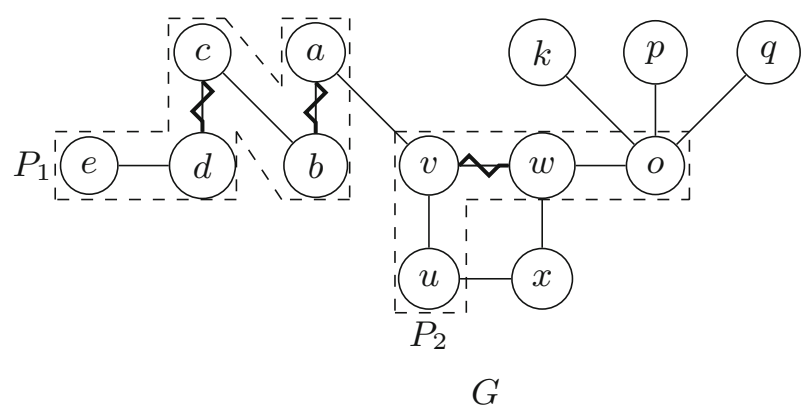

Fig. $6 M$-alternating path and $M$-augmenting path
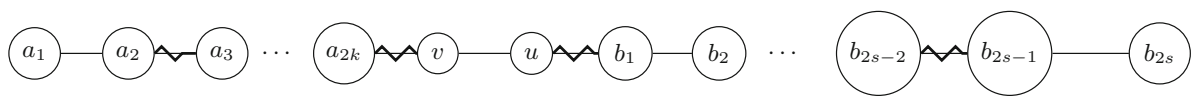

Fig. $7 \quad M$-augmenting path $P$

The following is a classic result, it characterizes maximum matchings in a graph $G$.

Lemma 5.2 (Berge 1957) A matching $M$ is maximum in $G$ if and only if $G$ does not have an $M$-augmenting path.

We now give a closed formula for the matching number of unicyclic graphs $G$ of Type I. This formula depends on the core and $N$-vertices of subtrees.

Theorem 5.3 If $G$ is a unicyclic graph of Type I and $G\{v\}$ its pendant tree such that $v \notin$ $\operatorname{Supp}(G\{v\})$, then

$$
v(G)=|\operatorname{Core}(G\{v\})|+|\operatorname{Core}(G-G\{v\})|+\frac{\left|V\left(\mathcal{F}_{N}(G\{v\})\right)\right|+\left|V\left(\mathcal{F}_{N}(G-G\{v\})\right)\right|}{2} .
$$

Proof Let $M_{1} \in \mathcal{M}(G\{v\})$ and $M_{2} \in \mathcal{M}(G-G\{v\})$. Let $u, w \in N(v) \cap V(G-G\{v\})$ and $M=M_{1} \cup M_{2}$. We will prove that $M \in \mathcal{M}(G)$. Suppose that $M \notin \mathcal{M}(G)$. Hence, by Lemma 5.2, there is an $M$-augmenting path, denoted by $P$. Notice that $P$ is neither totally contained in $G\{v\}$ nor totally contained in $G-G\{v\}$; otherwise, $P$ would be $M_{1}$ augmenting or $M_{2}$-augmenting, which is a contradiction, because $M_{1} \in \mathcal{M}(G\{v\})$ and $M_{2} \in \mathcal{M}(G-G\{v\})$, respectively. Moreover, $P$ does not contain the edges $\{u, v\}$ and the edge $\{w, v\}$ simultaneous, because $\{u, v\},\{w, v\} \notin M$. Then $P$ starts at a vertex $a_{1}$ of $G\{v\}$ and ends at a vertex $b_{2 s}$ of $G-G\{v\}$. Now we notice that the path $P$ contains the edge $\{u, v\}$ or the edge $\{w, v\}$. Suppose that $P$ contains the edge $\{u, v\}$, then we have $P=\left(a_{1}, a_{2}, \ldots, a_{2 k}, v, u, b_{1}, b_{2}, \ldots, b_{2 s}\right)$ (see Fig. 7).

Let $B_{1}=\left\{\left\{a_{2 k}, v\right\},\left\{u, b_{1}\right\}\right\} \cup \bigcup_{i=1}^{k-1}\left\{\left\{a_{2 i}, a_{2 i+1}\right\}\right\} \cup \bigcup_{j=1}^{s-1}\left\{\left\{b_{2 j}, b_{2 j+1}\right\}\right\}$ and $B_{2}=$ $\{\{u, v\}\} \cup \bigcup_{i=1}^{k}\left\{\left\{a_{2 i-1}, a_{2 i}\right\}\right\} \cup \bigcup_{j=1}^{s}\left\{\left\{b_{2 j-1}, b_{2 j}\right\}\right\}$. We observe that $B_{1} \subseteq M$ and $B_{2} \cap M=$ $\emptyset$. Let $M^{\prime}$ be a matching in $G$ given by $M^{\prime}=\left(M \cup B_{2}\right) \backslash B_{1}$. As $M^{\prime} \cap E(G\{v\})$ is a matching in $G\{v\}$, we see that it does not saturate $v$, because $v \notin \operatorname{Supp}(G\{v\})$. Hence, $M^{\prime} \cap E(G\{v\}) \notin \mathcal{M}(G\{v\})$ (see Lemma 2.7), that is, $\left|M^{\prime} \cap E(G\{v\})\right|<\left|M_{1}\right|$. We have

$$
\begin{aligned}
\left|M^{\prime} \cap E(G\{v\})\right| & =\left|\left(\left(M \cup B_{2}\right) \backslash B_{1}\right) \cap E(G\{v\})\right| \\
& =\left|\left(\left(M_{1} \cup M_{2} \cup B_{2}\right) \backslash B_{1}\right) \cap E(G\{v\})\right| \\
& =\left|\left(\left(M_{1} \backslash B_{1}\right) \cup\left(M_{2} \backslash B_{1}\right) \cup\left(B_{2} \backslash B_{1}\right)\right) \cap E(G\{v\})\right|
\end{aligned}
$$




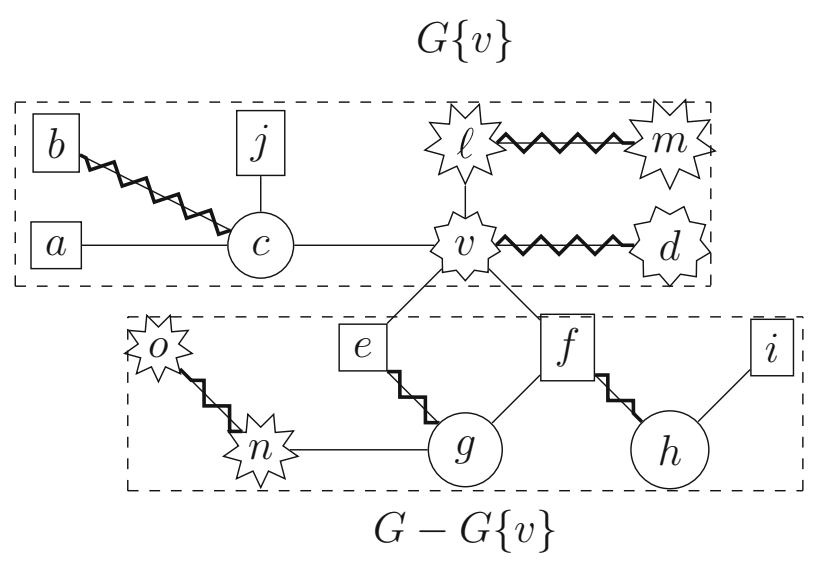

Fig. 8 Unicyclic graph of Type I and support of subtrees

$$
\begin{aligned}
& =\left|\left(\left(M_{1} \backslash B_{1}\right) \cup\left(M_{2} \backslash B_{1}\right) \cup B_{2}\right) \cap E(G\{v\})\right| \\
& =\left|\left(\left(M_{1} \backslash B_{1}\right) \cap E(G\{v\})\right) \cup\left(\left(M_{2} \backslash B_{1}\right) \cap E(G\{v\})\right) \cup\left(B_{2} \cap E(G\{v\})\right)\right| \\
& =\left|\left(\left(M_{1} \cap E(G\{v\})\right) \backslash B_{1}\right) \cup\left(\left(M_{2} \cap E(G\{v\})\right) \backslash B_{1}\right) \cup\left(B_{2} \cap E(G\{v\})\right)\right| \\
& =\left|\left(M_{1} \backslash B_{1}\right) \cup\left(B_{2} \cap E(G\{v\})\right)\right| \\
& =\left|\left(M_{1} \backslash B_{1}\right)\right|+\left|\left(B_{2} \cap E(G\{v\})\right)\right| \\
& =\left|M_{1}\right|-\left|M_{1} \cap B_{1}\right|+\left|\left(B_{2} \cap E(G\{v\})\right)\right| \\
& =\left|M_{1}\right|-\left|\left\{\left\{a_{2 k}, v\right\}\right\} \cup \bigcup_{i=1}^{k-1}\left\{\left\{a_{2 i}, a_{2 i+1}\right\}\right\}\right|+\left|\bigcup_{i=1}^{k}\left\{\left\{a_{2 i-1}, a_{2 i}\right\}\right\}\right| \\
& =\left|M_{1}\right|-k+k=\left|M_{1}\right| .
\end{aligned}
$$

By Eq. (3), we have $\left|M_{1}\right|>\left|M^{\prime} \cap E(G\{v\})\right|=\left|M_{1}\right|$, which is a contradiction.

The case where $P$ contains the edge $\{w, v\}$ is analogous. Therefore, $M \in \mathcal{M}(G)$. Using Lemma 4.3, we have

$$
\begin{gathered}
v(G\{v\})=|\operatorname{Core}(G\{v\})|+\frac{\left|V\left(\mathcal{F}_{N}(G\{v\})\right)\right|}{2} \text { and } \\
v(G-G\{v\})=|\operatorname{Core}(G-G\{v\})|+\frac{\left|V\left(\mathcal{F}_{N}(G-G\{v\})\right)\right|}{2} .
\end{gathered}
$$

Therefore, the matching number of $G$ is given by

$$
\begin{aligned}
v(G) & =\left|M_{1}\right|+\left|M_{2}\right|=v(G\{v\})+v(G-G\{v\}) \\
& =|\operatorname{Core}(G\{v\})|+|\operatorname{Core}(G-G\{v\})|+\frac{\left|V\left(\mathcal{F}_{N}(G\{v\})\right)\right|+\left|V\left(\mathcal{F}_{N}(G-G\{v\})\right)\right|}{2} .
\end{aligned}
$$

As an example of Theorem 5.3, consider the unicyclic graph $G$ of Fig. 8. To see that it is of Type I, we notice that $v \notin \operatorname{Supp}(G\{v\})=\{a, b, j\}$, then by Proposition 3.3, we have $G$ is a unicyclic graph of Type I. Moreover, $\operatorname{Supp}(G-G\{v\})=\{e, f, i\}, \operatorname{Core}(G\{v\})=\{c\}$, $V\left(\mathcal{F}_{N}(G\{v\})\right)=\{v, d, \ell, m\}$, Core $(G-G\{v\})=\{g, h\}$ and $V\left(\mathcal{F}_{N}(G-G\{v\})\right)=\{o, n\}$.

Therefore, by Theorem 5.3, we have that the matching number of $G$ is given by

$$
v(G)=|\operatorname{Core}(G\{v\})|+|\operatorname{Core}(G-G\{v\})|+\frac{\left|V\left(\mathcal{F}_{N}(G\{v\})\right)\right|+\left|V\left(\mathcal{F}_{N}(G-G\{v\})\right)\right|}{2}
$$




$$
=1+\frac{4}{2}+2+\frac{2}{2}=6
$$

We point out that $M=\{\{b, c\},\{v, d\},\{\ell, m\},\{n, o\},\{e, g\},\{f, h\}\}$ is a maximum matching of $G$ and $|M|=6$. II.

We now present a similar result for the matching number of unicyclic graphs $G$ of Type

Theorem 5.4 Let $G$ be a unicyclic graph and $C$ its cycle. Let $G-C=\bigcup_{i=1}^{k} T_{i}$, where $T_{i}$ is a connected component of $G-C$. If $G$ is a unicyclic graph of Type II, then

$$
v(G)=\left\lfloor\frac{|V(C)|}{2}\right\rfloor+\sum_{i=1}^{k}\left|\operatorname{Core}\left(T_{i}\right)\right|+\frac{\left|V\left(\mathcal{F}_{N}\left(T_{i}\right)\right)\right|}{2} .
$$

Proof For each pendant tree $G\{v\}$, with $v \in V(C)$, choose an $M_{v} \in \mathcal{M}(G\{v\})$ that does not saturate $v$. Note that this maximum matching exists because $G$ is a unicyclic graph of Type II, and this implies that $v \in \operatorname{Supp}(G\{v\})$ for all $v \in V(C)$. Hence, by Lemma 2.7, we have that $M_{v}$ exists. Choose an $M_{c} \in \mathcal{M}(C)$ and let $M=M_{c} \cup\left(\bigcup_{v} M_{v}\right)$.

We will show that $M \in \mathcal{M}(G)$. Suppose by contradiction that $M \notin \mathcal{M}(G)$. Then, by Lemma 5.2, there exists a $M$-augmenting path denoted by $P$ in $G$. Note that if $V(P) \subseteq V(C)$, then $P$ would be a $M_{c}$-augmenting path in $C$ which is a contradiction because $M_{C} \in \mathcal{M}(C)$. Now, if $V(P) \subseteq V(G\{v\})$, then $P$ would be an $M_{v}$-augmenting path in $G\{v\}$, which is a contradiction because $M_{v} \in \mathcal{M}(G\{v\})$.

Note that the only way to obtain an $M$-augmenting path $P$ in $G$ is if we start the path $P$ at a vertex $u \in V(G\{v\})$, with $u \neq v$, and end at a vertex $z \notin V(G\{v\})$. If that happens, there would be an $M_{v}$-alternating path starting in $u$ and ending in $v$ contained in $P$. But since $M_{v}$ does not saturate $v$, actually we would obtain a $M_{v}$-augmenting path in $G\{v\}$, which is a contradiction, because $M_{v} \in \mathcal{M}(G\{v\})$. Therefore, $M \in \mathcal{M}(G)$.

By Lemma 4.3, we have

$$
v\left(T_{i}\right)=\left|\operatorname{Core}\left(T_{i}\right)\right|+\frac{\left|V\left(\mathcal{F}_{N}\left(T_{i}\right)\right)\right|}{2} .
$$

Moreover, we have $v(C)=\left\lfloor\frac{|V(C)|}{2}\right\rfloor$. Since $v$ is not saturated by $M_{v}$ in $G\{v\}$, we see that $M_{v} \in \mathcal{M}(G\{v\}-v)$, that is, $v(G\{v\})=v(G\{v\}-v)$. Therefore, we have that the matching number is given by

$$
\begin{aligned}
v(G) & =\left|M_{C}\right|+\sum_{v \in V(C)}\left|M_{v}\right|=v(C)+\sum_{v \in V(C)} v(G\{v\}) \\
& =v(C)+\sum_{v \in V(C)} v(G\{v\}-v)=v(C)+\sum_{i=1}^{k} v\left(T_{i}\right) \\
& =\left\lfloor\frac{|V(C)|}{2}\right\rfloor+\sum_{i=1}^{k}\left|\operatorname{Core}\left(T_{i}\right)\right|+\frac{\left|V\left(\mathcal{F}_{N}\left(T_{i}\right)\right)\right|}{2} .
\end{aligned}
$$

Consider $G$ the unicyclic graph of Fig. 9. We see that $G$ is a unicyclic graph of Type II, because $a \in \operatorname{Supp}(G\{a\})=\{a, j, l, m\}, b \in \operatorname{Supp}(G\{b\})=\{b, h, g\}, c \in$ $\operatorname{Supp}(G\{c\})=\{c, o\}, d \in \operatorname{Supp}(G\{d\})=\{d, u, v, w, t\}$ and $e \in \operatorname{Supp}(G\{e\})=\{e\}$. By Corollary 3.4, we have that $G$ is of Type II. Notice that $G-C_{5}=\bigcup_{i=1}^{4} T_{i}$, where 

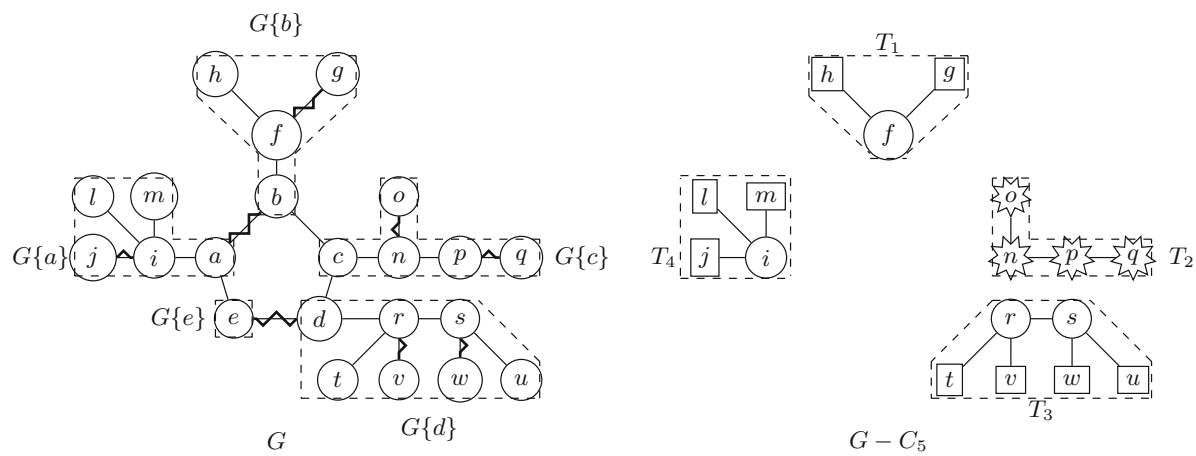

Fig. 9 Unicyclic graph of Type II and its subtrees $T_{1}, T_{2}, T_{3}$ and $T_{4}$

Table 2 Support, core and $N$-vertices of the trees $T_{1}, T_{2}, T_{3}$ and $T_{4}$

\begin{tabular}{lll}
\hline Support & Core & $N$-vertices \\
\hline $\operatorname{Supp}\left(T_{1}\right)=\{h, g\}$ & $\operatorname{Core}\left(T_{1}\right)=\{f\}$ & $V\left(\mathcal{F}_{N}\left(T_{1}\right)\right)=\emptyset$ \\
$\operatorname{Supp}\left(T_{2}\right)=\emptyset$ & $\operatorname{Core}\left(T_{2}\right)=\emptyset$ & $V\left(\mathcal{F}_{N}\left(T_{2}\right)\right)=\{n, o, p, q\}$ \\
$\operatorname{Supp}\left(T_{3}\right)=\{t, v, u, w\}$ & $\operatorname{Core}\left(T_{3}\right)=\{r, s\}$ & $\left.V\left(\mathcal{F}_{N}\left(T_{3}\right)\right)\right)=\emptyset$ \\
$\operatorname{Supp}\left(T_{4}\right)=\{j, l, m\}$ & $\left.\operatorname{Core}\left(T_{4}\right)\right)=\{i\}$ & $V\left(\mathcal{F}_{N}\left(T_{4}\right)\right)=\emptyset$ \\
\hline
\end{tabular}

$T_{1}=G[\{f, g, h\}], T_{2}=G[\{n, o, p, q\}], T_{3}=G[\{r, s, t, u, v, w\}]$ and $T_{4}=G[\{j, i, l, m\}]$ (see Fig. 9). We see that $T_{2}$ has perfect matching, then $T_{2}$ is non singular and so $T_{2}$ has empty support. Moreover, we have that $T_{1}, T_{3}$ and $T_{4}$ do not have perfect matchings, then they are singular and their supports are given in Table 2.

Therefore, by Theorem 5.4, we have that the matching number of $G$ is given by

$$
\begin{aligned}
v(G) & =\left\lfloor\frac{|V(C)|}{2}\right\rfloor+\sum_{i=1}^{k}\left|\operatorname{Core}\left(T_{i}\right)\right|+\frac{\left|V\left(\mathcal{F}_{N}\left(T_{i}\right)\right)\right|}{2} . \\
& =\left\lfloor\frac{5}{2}\right\rfloor+1+\frac{4}{2}+2+1=8 .
\end{aligned}
$$

We point out that $M=\{\{a, b\},\{d, e\},\{i, j\},\{f, g\},\{p, q\},\{n, o\},\{r, v\},\{s, w\}\}$ is a maximum matching of $G$ and $|M|=8$.

Notice that, once we have the cycle and the pendant trees of a unicyclic graph $G$, according to Proposition 3.3 and Corollary 3.4, we can decide if $G$ is Type I or Type II computing the support of each pendant tree in polynomial time. Therefore, the closed formulas of Theorems 4.8, 4.10, 5.3 and 5.4 are of practical use.

Acknowledgements Work supported by MATHAMSUD 18-MATH-01. Maikon Toledo thanks CAPES for their support. V. Trevisan acknowledges partial support of CNPq Grants 409746/2016-9 and 303334/2016-9, and FAPERGS (Proj. PqG 17/2551-0001).

\section{References}

Alon N, Boppana RB (1987) The monotone circuit complexity of Boolean functions. Combinatorica 7:1-22 
Alon N, Kahale N (1998) Approximating the independence number via the J-function. Math Program 80:253264

Berge C (1957) Two theorems in graph theory. Proc Natl Acad Sci 43(9):842-844

Bevis JH, Domke GS, Miller VA (1995) Ranks of trees and grid graphs. J Combin Math Combin Comput 18:109-119

Cvetkovć DM, Doob M, Sachs H (1980) Spectra of graphs: theory and application, vol 87. Academic Press, London

Frieze AM (1990) On the independence number of random graphs. Discrete Math 81:171-175

Gong S, Fan Y, Yin Z (2010) On the nullity of graphs with pendant trees. Linear Algebra Appl 433:1374-1380

Jaume DA, Molina G (2018) Null decomposition of trees. Discrete Math 341:836-850

Karp RM (1972) Reducibility among combinatorial problems. In: Miller RE, Thatcher JW, Bohlinger JD (eds) Complexity of computer computations. The IBM Research Symposia Series. Springer, Berlin, pp 85-103

König D (1916) Gráfok és alkalmazásuk a determinánsok és a halmazok. Matematikai śs Természettudományi Értesítö 34:104-119

Ming GJ, Wang TS (2001) A relation between the matching number and laplacian spectrum of a graph. Linear Algebra Appl 325:71-74

Shearer JB (1983) A note on the independence number of triangle-free graphs. Discrete Math 46:83-87

Sookyang S, Arworn S, Wojtylak P (2008) Characterizations of non-singular cycles, path and trees. Thai J Math 6:331-336

Publisher's Note Springer Nature remains neutral with regard to jurisdictional claims in published maps and institutional affiliations. 